\title{
References and Citations: Is It Time to Update Their Format?
}

\author{
Sai Krishna Gudi (iD ${ }^{1,{ }^{*}}$, Komal Krishna Tiwari (iD ${ }^{2}$ and Rahamthulla Shaik ${ }^{3}$ \\ ${ }^{1}$ College of Pharmacy, Rady Faculty of Health Sciences, University of Manitoba, Winnipeg, Canada \\ ${ }^{2}$ Department of Occupational Therapy, College of Rehabilitation Sciences, University of Manitoba, Winnipeg, Canada \\ ${ }^{3}$ Department of Pharmacy Practice, Chebrolu Hanumaiah Institute of Pharmaceutical Sciences, Andhra Pradesh, India \\ "Corresponding author: College of Pharmacy, Rady Faculty of Health Sciences, University of Manitoba, Winnipeg, Canada. Email: gudisk@myumanitoba.ca
}

Received 2021 May 21; Revised 2021 June 09; Accepted 2021 June 21.

Keywords: Citation, Journal, Publication, Reference, Science

\section{Dear editor,}

This letter highlights two critical issues around referencing and citations along with their consequences, and the possible solutions. In science, referencing is an imperative way of acknowledging ideas originally derived from other sources or authors $(1,2)$. Besides, a reference provides information necessary for readers to track the original source referred in the article (1). In lay terms, the process of directing the readers to the different sources of information supporting an idea, argument or concept, and acknowledging the authors is known as referencing. Improper or inappropriate reference leads to various concerns and could abate the quality of the entire work. Thus, cross-checking whether the cited reference is accurate, and locating the actual content in the original source document is imperative.

When it comes to scholarly writing and publishing, accuracy plays a vital role. The act of looking up a reference for verification serves as a checkpoint of appropriateness, where referencing acts as a fact-checking tool (3). However, improper attribution of references might occasionally go unchecked. Accordingly, citation is a common and essential practice in scientific writing that offers credit to authors for their unconventional work that another researcher uses in supporting their claim $(2,4)$. However, regardless of its significance, the citation may be the least noticed aspect of a scientific manuscript (5). Given this background, the authors would like to highlight two important issues and their consequences, and the possible solutions that are overlooked when quoting a reference and a citation.

The first issue is the perplexity in identifying the exact content (a sentence or a paragraph) in a scientific article to which the reference is directed to. Although there exist numerous referencing styles, quoting a reference and a cita- tion remains analogous. Thus, usually, the citation within the text is quoted as a superscript or within the line in the parenthesis, for which further details of the citation will be provided in the reference section. It is often a tedious task to track where exactly that particular citation is referring to in the original source article, especially when the source article is too lengthy such as a protocol or a guideline. Hence, it is a laborious job for a reader to cross-check the original source for which the reference has been cited, and as a result, the majority of research works are left underrated or unrecognized. The possible solution to overcome this problem is to make the reader walk through the original source article in identifying where exactly the citation is re-directed to. This problem could be addressed by adjoining a line or paragraph number (if possible, a page number) within parentheses as an extension to the existing referencing styles.

Practical Illustration: Statement- "Not all dairy products exhibit a beneficial role in preventing type-2 diabetes, where only low-fat dairy foods such as yogurt have a favorable role, while most other dairy foods show no association".

Suggested citation- Gudi SK. Dairy consumption and risk of type-2 diabetes: The untold story. Ann Pediatr Endocrinol Metab. 2021; 26(1):14-18. (page 3, line 96).

The second issue is the unvariedness in citation credits for authors whose work has been cited multiple times within the same article. Citations play a pivotal role in assessing the worthiness of an article and the author, eventually. However, regardless of the number of times a particular article is cited in the paper, the researcher receives only one citation. For example, reference A has been cited multiple times within the same article, whereas reference B has been cited only once in the entire article. At last, both these references (or authors) get only one citation as a credit, al- 
though reference A has been cited multiple times to support multiple ideas within the same article. Albeit quoting the reference multiple times, the existing referencing system cannot differentiate the citations based on the number of times cited, leaving little space in assessing the author's impact. The possible solution to address this issue is to adjoin this piece of information (i.e., the number of times a particular reference has been cited within the same article) to the existing referencing style, which will help understand the concept of times cited within the same article.

Practical Illustration: Suggested citation- Gudi SK. Eating speed and the risk of type 2 diabetes: explorations based on real-world evidence. Ann Pediatr Endocrinol Metab. 2020;25(2):80-83. (Times cited-3).

In this rapidly evolving digital age of publishing, citations and references play a vital role in hypothesis generation and sourcing the original work (6). Citations and references provide a means of acknowledging the previous work, enabling new studies to integrate within the existing literature, and identify primary sources supporting the authors' statements. Thus, being accurate and transparent while citing and referencing allows readers to follow the flow of ideas that ultimately enhance science communication integrity (7).

In conclusion, it is a tedious task to look through the entire original source in locating the exact sentence or a paragraph that the reference directs the reader in an article. Thus, mentioning the line or a paragraph number in the existing referencing system will help the reader find out the referenced article's information and save readers' time. Equality in allocating the citation credits of the articles that are cited multiple times within the same publication is another issue, where adding this information (i.e., times cited) as a suffix to a reference helps the authors ac- quire the citation credits accordingly.

\section{Footnotes}

Authors' Contribution: Study concept and design: SKG, KKT, and RS. Drafting of the manuscript: SKG and RS. Critical revision of the manuscript for important intellectual content: SKG and KKT. Approval of the final version of the manuscript: SKG, KKT, and RS.

Conflict of Interests: The authors have no conflict of interest to declare.

Funding/Support: The authors received no funding or support towards this work.

\section{References}

1. Mack C. The Editorial Review Process. J Micro/Nanolithogr MEMS MOEMS. 2015;14(3). doi: 10.1117/1.jmm.14.3.030101.

2. Bahadoran Z, Mirmiran P, Kashfi K, Ghasemi A. The Principles of Biomedical Scientific Writing: Citation. Int J Endocrinol Metab. 2020;18(2). e102622. doi: 10.5812/ijem.102622. [PubMed: 32636888]. [PubMed Central: PMC7322669].

3. Barroga EF. Reference accuracy: authors', reviewers', editors', and publishers' contributions. J Korean Med Sci. 2014;29(12):1587-9. doi: 10.3346/jkms.2014.29.12.1587. [PubMed: 25469055]. [PubMed Central: PMC4248576].

4. Lingard L. Writing an effective literature review : Part II: Citation technique. Perspect Med Educ. 2018;7(2):133-5. doi: 10.1007/s40037-0180407-z. [PubMed: 29500746]. [PubMed Central: PMC5889378].

5. Mertens S, Baethge $C$. The virtues of correct citation: careful referencing is important but is often neglected/even in peer reviewed articles. Dtsch Arztebl Int. 2011;108(33):550-2. doi: 10.3238/arztebl.2011.0550. [PubMed: 21912574]. [PubMed Central: PMC3167934].

6. Gudi SK, Basker SP. Self-promotions and advertising: are they a common practice for boosting altmetric scores? Sci Edit. 2019;6(2):151-3. doi: $10.6087 /$ kcse.177.

7. Bryson D. Using research papers: citations, referencing and plagiarism. J Vis Commun Med. 2012;35(2):82-4. doi: 10.3109/17453054.2012.690128. [PubMed: 22747269]. 